\title{
Efficacy of Tuina in patients with chronic low back pain: study protocol for a randomized controlled trial
}

Shuaipan Zhang ${ }^{1,2+}$, Lingjun Kong ${ }^{2+}$, Qingguang Zhu ${ }^{2}$, Zhiwei Wu ${ }^{1,2}$, Jianhua $\mathrm{Li}^{1}$, Min Fang ${ }^{2,3^{*}}$, Wuquan Sun ${ }^{1}$, Yanbin Cheng' ${ }^{2}$, Shanda Xu ${ }^{1,2}$, Guangxin Guo ${ }^{1,2}$, Xin Zhou ${ }^{1,2}$ and Zhizhen Lv $v^{1,2}$

\begin{abstract}
Background: Low back pain is a common reason for medical care and carries a heavy social burden. The efficacy of Tuina or health care education for low back pain has been evaluated in previous systematic reviews. However, there is no evidence to support the superiority of one form of treatment over another. The aim of this study is to compare the efficacy of Tuina with health care education in the management of low back pain.

Methods/design: This study is a randomized controlled trial with parallel-group design including two groups: a Tuina group and a health care education group. A total of 160 eligible participants will be randomly assigned to the groups in a 1:1 ratio. The interventions of both groups will last for 20 min and be carried out twice each week for a period of 12 weeks. The primary outcome is the Oswestry Disability Index. The secondary outcomes include a visual analogue scale and the 36-item Short Form Health Survey. They will be assessed at baseline, at the end of the intervention every month, and during 6 months and 9 months of follow-up by repeated measures analysis of variance. The significance level is $5 \%$. The safety of Tuina and health care education will be evaluated after each treatment session. This study will focus on the value of Tuina and health care education for low back pain and will highlight any differences in the efficacy of the treatments.
\end{abstract}

Discussion: This study will evaluate the efficacy and safety of Tuina intervention for low back pain, which could provide reliable evidence for clinical decision making for patients with low back pain.

Trial registration: Chinese Clinical Trial Registry, ChiCTR1900022656. Registered on 23 April 2019.

Keywords: Low back pain, Efficacy, Randomized controlled trial, Tuina, Health care education

\section{Background}

Low back pain is a common symptom in today's society. It causes serious health and economic burdens. A study showed that the health cost of low back pain was increasing in every year, and the number of persons with disability

\footnotetext{
*Correspondence: fangmin19650510@163.com

†Shuaipan Zhang and Lingjun Kong contributed equally to this work. ${ }^{2}$ Institute of Tuina, Shanghai Institute of Traditional Chinese Medicine, Shanghai 200437, China

${ }^{3}$ School of Acupuncture-Moxibustion and Tuina, Shanghai University of Traditional Chinese Medicine, Shanghai 201203, China

Full list of author information is available at the end of the article
}

due to low back pain increased by $54 \%$ between 1990 and 2015 [1]. In high-income countries, the highest point prevalence of low back pain has reached 32.9\% [2]. According to the epidemiologic data of low back pain in Asia, it was defined as a health problem associated with functional limitations and financial burden [3, 4].

Low back pain can be attributed to excessive physical exertion or trauma, resulting in damage or degradation of the vertebrae, intervertebral discs, or spinal muscles. Now, we focus more on nonspecific low back pain, which typically can account for $90 \%$ of the patients [5]. The duration

(C) The Author(s). 2020 Open Access This article is licensed under a Creative Commons Attribution 4.0 International License, which permits use, sharing, adaptation, distribution and reproduction in any medium or format, as long as you give appropriate credit to the original author(s) and the source, provide a link to the Creative Commons licence, and indicate if changes were made. The images or other third party material in this article are included in the article's Creative Commons licence, unless indicated otherwise in a credit line to the material. If material is not included in the article's Creative Commons licence and your intended use is not permitted by statutory regulation or exceeds the permitted use, you will need to obtain permission directly from the copyright holder. To view a copy of this licence, visit http://creativecommons.org/licenses/by/4.0/. The Creative Commons Public Domain Dedication waiver (http://creativecommons.org/publicdomain/zero/1.0/) applies to the data made available in this article, unless otherwise stated in a credit line to the data. 
of pain persists for at least 3 months and must meet the International Classification of Diseases, 11th Revision (ICD11), criteria for chronic primary pain [6]. Owing to the considerable pain and cost associated with chronic pain, some interventions must be applied to prevent its exacerbation [7].

A treatment option for individuals with low back pain is a risk stratification approach depending on screening tools such as the Orebro Musculoskeletal Pain Screening Questionnaire [8] and the STarTBack tool [9], which aim to limit progression to chronic low back pain. With a high risk, regardless of the potential harm with some pain medicines, opioids [10] and nonsteroidal anti-inflammatory drugs [11] will be used to control the condition when it does not respond to nonpharmacological treatments [12]. Surgery will also be used to relieve the compression of nerve roots caused by degenerative disc disease [13]. However, some guidelines have endorsed the cautious use of medication and surgery and take nonpharmacological and noninvasive treatments as a first-line treatment, including routine health education, exercise, psychotherapy, and physical therapies, owing to the risk of trauma and the cost [14-17]. A relatively new and promising approach in the health care education of low back pain has focused on the neurobiology and neurophysiology of pain, which attempts to help the patients understand that pain and tissue injury are different constructs $[18,19]$. In addition, education is composed of the factors that affect the occurrence of the disease, including weight, occupation, and exercise style; explanation of the natural development process and harm of the disease; and explanation of the planning and prognosis of disease treatment. For patients with chronic low back pain, several studies have shown favorable outcomes following health care education in patients with chronic pain in terms of pain relief and improved physical performance [20-22]. It can also reduce emotional distress and subsequent health care use [23, 24].

Additionally, some systematic reviews showed a moderate level of evidence for the clinical efficacy of manual therapy, massage, and spinal manipulations in patients with low back pain [25-27]. Tuina, one of the key noninvasive therapies of traditional Chinese medicine, is a Chinese manual manipulation. It has been used for thousands of years in China. Some studies showed the potential effect of Tuina for chronic neck pain [28], but more clinical trials are required to provide evidence of Tuina for low back pain. Health care education has also shown promise in previous reviews to improve pain and disability in chronic low back pain [29, 30]. Therefore, this study was designed to compare the efficacy of Tuina with health care education for patients with low back pain.

\section{Methods/design}

\section{Study design}

This study is a single-center, assessor- and analystblinded randomized controlled trial conducted in Shanghai, China, at Yueyang Hospital of Integrated Traditional Chinese and Western Medicine affiliated with Shanghai University of Traditional Chinese Medicine. In total, 160 patients will be recruited and randomly assigned to a Tuina group and a health care education group in a 1:1 ratio. This trial protocol was approved by the Ethics Committee of Yueyang Hospital of Integrated Traditional Chinese and Western Medicine affiliated with Shanghai University of Traditional Chinese Medicine (project number 2019-035). It is registered with the Chinese Clinical Trial Registry (ChiCTR1900022656). Written informed consent will be provided by all patients before the screening. The interventions will be given twice per week for 3 months. We will ask some researchers who are blinded to assignment to accomplish the outcome assignment and statistical analyses independently. The trial flowchart and study design are shown in Figs. 1 and 2, respectively.

\section{Participant recruitment}

Patients with chronic low back pain consistent with the disease definition in the ICD-11 will be recruited. The participants are outpatients and inpatients in Tuina departments of Yueyang Hospital of Integrated Traditional Chinese and Western Medicine affiliated with Shanghai University of Traditional Chinese Medicine. We will also seek potential patients through posters, Internet advertisements, and the official microblog and WeChat platforms. All participants are aged 18 to 65 years. We define 10 years as an interval, and the number of participants in each interval is almost equal.

\section{Inclusion criteria}

The inclusion criteria are as follows:

1. Duration of low back pain as the main symptom for at least 3 months

2. Pain lasting more 20 min per time and at least once per month

3. Aged between 18 and 65 years, male or female

4. Volunteer to take part in the study and sign the informed consent form

5. Promise not to receive other related therapy during the period of treatment

6. Capable of complying with the intervention and follow-up assessment

\section{Exclusion criteria}

The exclusion criteria are as follows: 


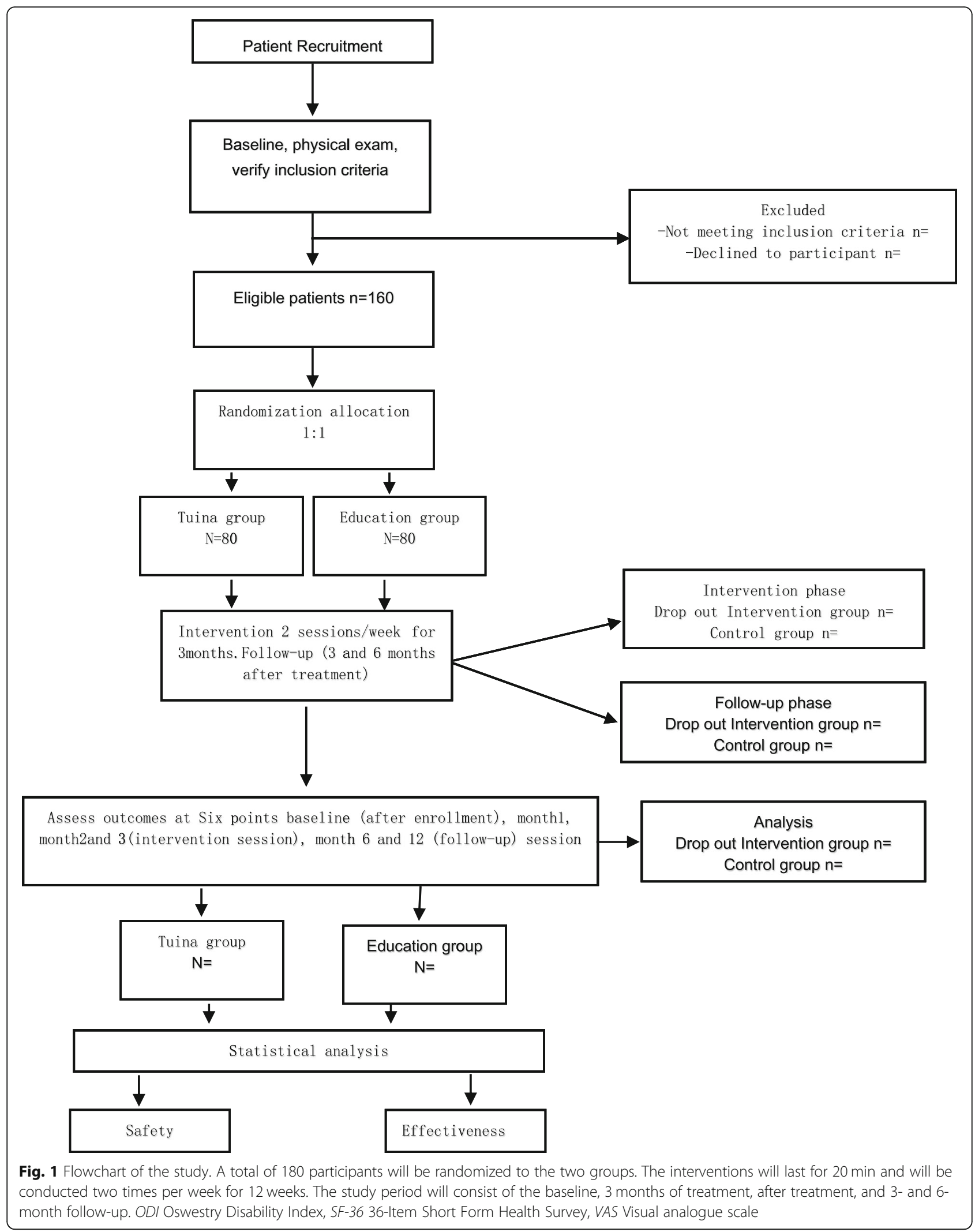




\begin{tabular}{|c|c|c|c|c|c|c|c|}
\hline Time & $M-1$ & MO & M1 & M2 & M3 & M6 & M9 \\
\hline Period & Screening & Baseline & \multicolumn{3}{|c|}{ Intervention } & \multicolumn{2}{|c|}{ Follow-up } \\
\hline \multicolumn{8}{|l|}{ Patients } \\
\hline Eligibility & $x$ & & & & & & \\
\hline Demography & $x$ & & & & & & \\
\hline Informed consent & $x$ & & & & & & \\
\hline \multicolumn{2}{|l|}{ Sign the informed consent } & $x$ & & & & & \\
\hline Medical history & $x$ & & $\mathrm{x}$ & $x$ & $x$ & $x$ & $\mathrm{x}$ \\
\hline Physical examination & $x$ & & $x$ & $x$ & $x$ & $x$ & $\mathrm{x}$ \\
\hline Randomization & & $\mathrm{x}$ & & & & & \\
\hline \multicolumn{8}{|l|}{ intervention } \\
\hline Tuina group $(n=80)$ & & & \multicolumn{5}{|c|}{24 sessions of tuina } \\
\hline Education group $(\mathrm{n}=80$ & & & \multicolumn{5}{|c|}{24 sessions of education } \\
\hline \multicolumn{8}{|l|}{ Outcomes } \\
\hline ODI & & $x$ & $x$ & $x$ & $\mathrm{x}$ & $x$ & $\mathrm{x}$ \\
\hline VAS & & $x$ & $\mathrm{x}$ & $x$ & $x$ & $\mathrm{x}$ & $\mathrm{x}$ \\
\hline SF-36 & & $\mathrm{x}$ & $x$ & $x$ & $x$ & $x$ & $\mathrm{x}$ \\
\hline \multicolumn{8}{|l|}{ Trail evaluation } \\
\hline Patient's compliance & & & $x$ & $x$ & $\mathrm{x}$ & $x$ & $x$ \\
\hline Safety evaluation & & & $x$ & $x$ & $x$ & $x$ & $\mathrm{x}$ \\
\hline Credibility test & & & $x$ & $x$ & $x$ & $x$ & $\mathrm{x}$ \\
\hline Adverse events & & & $x$ & $x$ & $x$ & $x$ & $x$ \\
\hline Analysis & $x$ & $\mathrm{x}$ & $x$ & $x$ & $x$ & $x$ & $x$ \\
\hline
\end{tabular}

Fig. 2 Study schedule 
1. Chronic low back pain caused by local disease (e.g., lumbar fracture, lumbar tumor, lumbar tuberculosis, lumbar spine surgery or trauma)

2. Sciatica, myelopathy, displacement, or radiculopathy due to lumbar intervertebral disc disorders or spondylolisthesis

3. Severe primary disease such as cardiovascular, lung, kidney, and hematopoietic disease

4. Ongoing treatment within 5 days prior to inclusion

5. Mental disorder

6. Pregnant woman

7. Patients not suitable for nuclear magnetic resonance examination

\section{Dropout and suspension criteria}

Patients have the right to withdraw for any reason during the study period according to the Patient Management Protection Rules. The following conditions are considered as withdrawal criteria: (1) patient does not implement the protocol treatment on schedule; (2) participation in other treatments during the trial; (3) intolerable adverse events; and (4) lost to follow-up. In addition, if severe poor efficacy or adverse reactions occur during the trial, the trial will be forcibly suspended immediately.

\section{Randomization}

The randomization sequence will be generated by a random number generator (IBM SPSS Statistics version 21.0 software; IBM Corp., Armonk, NY, USA), which will be sent to a therapist with an opaque envelope numbered sequentially. The therapist will sequentially open random-allocation envelopes and allocate the participants accordingly. Eligible patients will be randomized to the Tuina group or the health care education group, with 80 patients in each group.

\section{Blinding}

Participants and therapists will not be blinded to treatment allocation, owing to the limitation of the therapies. Interventionists will be masked to the participants' outcome measurements. The evaluators, data managers, and statisticians will be blinded to the group allocation in the outcome evaluation procedure and data analysis for the sake of reducing the risk of bias.

\section{Interventions}

The participants will receive a total of 24 treatments in 3 months. The therapists must have 10 years of experience with Tuina treatment or be skillful in health care education for the participants. In addition, they are required to have passed a clinical test to make sure that the consistency of the trial is administered. It should be emphasized that some other treatments for low back pain during the trial will be forbidden, which includes medication therapy, surgery, drug injections, acupuncture, moxibustion, and physical therapy. If the subjects receive any other treatment, it will be recorded the changeable each time he arrives.

\section{Tuina group}

The tendons and bones theory of traditional Chinese medicine summarizes the physiology and pathology of physical movement. The relationship between tendons and bones in the human body is inseparable. During body movement, the muscles will contract to produce force and transfer it to the bone with the help of ligaments or tendons. At the same time, the bone can efficiently process the force to different parts of ligaments and tendons, resulting in a coordinated motion pattern. Coordination of tendons and bones is the main basis for maintaining the dynamic balance of the spine and joints of the body. Tuina of "restrict bone and harmonize joints" intends to restore the harmonious relationship between tendons and bones by manipulation of the low back with the therapist's hands. The specific process is outlined in the subsections below.

\section{Step 1: local manipulation}

First, the patients are in a prone position. The doctor stands on the side of the patient with the method of rolling the Bladder meridians on both sides of the spine, which is applied for 3 to $5 \mathrm{~min}$. The waist will be focused on to manipulate preferentially and then using the same technique on the patient's buttocks and lower limbs at the same time. It plays the role of relaxing sinews and activating collaterals with the theory of traditional Chinese medicine. Second, the physician will perform the manipulation on the patient's waist and the posterolateral side of the lower limbs by pressing, kneading, plucking, and so forth for 5 to $7 \mathrm{~min}$, which can relieve the cramping of the muscles. The doctors press the acupoints, including Mingmen (DU04), Shenshu (BL23), Yaoyangguan (DU03), Huantiao (GB30), Weizhong (BL40), Chengshan (BL57), and Ashi points, with the elbow or the thumb to achieve Deqi sensation, which is commonly regarded as an indicator of manipulation efficacy [31-34]. Last, scrubbing manipulation will be used to dilate the heat with the palms. In conclusion, the above content refers to therapeutic manipulation for sinew injury.

\section{Step 2: lumbar structural rectification}

Pulling manipulation on the lumbar vertebrae will be used to regulate the pathological condition of the disorder of the facet joint after finishing the relaxed manipulation [35]. First, the patient is required to face the doctor on the side of the bed in a lateral position. The lower limb in the upper part is on the knee, and the other lower limb is naturally straight. The physician stands on the ventral side of the patient, with elbow or hand against the front of his 
shoulder and the other elbow against the buttocks. The doctor will coordinate the strength with two hands or elbows, which begins with a small amount of rotation of the waist to relax the waist of the patient. Then, therapists gradually increase the angle of rotation of the patient's waist with relative force until the maximum extent. It usually ends up with the sound of a "click," which is without special pursuit during the clinical treatment. The same technique is applied to the contralateral lumbar vertebrae.

\section{Health care education group}

The education intervention will be given to the participants with two sessions per week. The first health education session is a group meeting including all of the participants in the control group using a PowerPoint presentation, which will last 30-60 min. An educational booklet and a video will be given to the patients to do the home-based online e-learning module, containing the same content provided in the PowerPoint presentation. The second session will be conducted as one-to-one communication focused on personal needs to guide patients to implement the key education in their lives. This intervention will be maintained by an experienced physical therapist for 12 weeks. The program will include the following two broad components: (1) introduce key concepts of pain biology and (2) present specification of recovery.

Each patient will be given an introduction about the underlying mechanisms, predisposing factors, and prognosis of low back pain. Pain will be explained as the conscious part of the response, which can be influenced by many factors as a protective output. Interventionists will formulate specification of daily routines for patients, for example: keep the back warm; keep a healthy diet and prevent obesity; maintain beneficial posture and abandon bad habitual posture, keeping a normal physiological curvature of the spine; avoid overwork, intense activity, or lifting heavy weights; maintain proper exercise; and maintain a good mental state and be prepared to endure chronic pain patiently for a long time.

\section{Outcome measurements}

The outcome will be measured by three self-report questionnaires, which can reflect the lumbar dysfunction, pain, and quality of life. Six time points will be used to assess outcomes, including baseline (after enrollment); months 1, 2, and 3 (intervention session); and months 6 and 9 (follow-up sessions).

\section{Primary outcome measurement Oswestry Disability Index}

The primary outcome is the subject's perceived disability, which is assessed by the modified Oswestry Disability Index (ODI) [36]. The ODI consists of ten items, including pain intensity, personal care, lifting, walking, sitting, standing, sleep, sex life, social life, and traveling. According to the extent of disability, each item is scored from 0 to 5 . The total score is reported as a percentage, which is actual score $/ 50$ (maximum possible score) $\times 100 \%$. The ODI results will be assessed using a repeated longitudinal analysis.

\section{Secondary outcome measurement \\ Visual analogue scale}

A visual analogue scale (VAS) is a 10-point scale selected to quantitatively measure the level of low back pain during the study, for which 0 means "none of pain" while 10 represent "the unbearable pain [37]". It has been proved to have validity and reliability for outcome measurement. We will assess the VAS with a repeated longitudinal analysis.

\section{Short-Form Health Survey}

The association between health-related quality of life and various factors will be assessed by using the 36-item Short Form Health Survey (SF-36), which includes 36 questions divided into 9 areas. It consists of vitality (4 items), physical functioning (10 items), bodily pain (2 items), general health (5 items), physical role (4 items), emotional role (3 items), social functioning (3 items), mental health (5 items), and health transition (5 items). For each field, scores range from 0 to 100 , with higher scores reflecting a better quality of life. A repeated longitudinal analysis will also be used to evaluate the SF-36.

\section{Safety evaluation}

Adverse events refer to the unexpected responses that occur during or after treatment, which can lead to a hospitalization or even threaten life. The trial should be suspended and immediate process is indispensable whenever we encounter adverse events. Also, we should record the details in the case report forms (CRFs). The efficacy and safety of the intervention will be evaluated with 6 months of unsupervised follow-up, during which the subjects should only be received routine lumbar care. At month 6 and 9, participants will be contacted with the phone for their physical condition of low back pain currently. In addition, evaluators can be reformed 13 with the clinical symptoms and AEs whenever the patients meet something important.

\section{Data collection and monitoring}

The screeners will collect data on the baseline characteristics when the patients are recruited, and CRFs will be completed with the outcome measurement by the assessor. This consists of questionnaire-based assessment of treatment effects, lumbar physiological function, adverse events, and safety evaluations. Then two data administrators, who are beyond the research team and blinded 
to group allocation, will independently receive the completed CRFs and enter them into an Excel database (Microsoft, Redmond, WA, USA). They are required to have completed rigorous training for the data monitoring. Then they will entry the real-time data in the Chinese Clinical Trial Registration Center, in which the electronic data management system will be used to track and monitor the test data in real-time in the Department of Science and Technology in Yueyang Hospital.

\section{Statistical analyses}

The intention-to-treat principle will be followed in the primary data analysis with IBM SPSS Statistics for Windows version 21.0 software by statisticians who are blinded to the group allocation. The baseline characteristics will be expressed with descriptive statistics for the two groups, which are reported as the mean \pm standard deviation. A Kolmogorov-Smirnov test with Lilliefors correction will be used to analyze all quantitative variables to determine whether they follow a normal distribution. Parametric statistics (Tukey test) or nonparametric statistics (Wilcoxon rank-sum test) will be used for the withinand between-group analyses in accordance with the results of the homogeneity and normality analyses. When initial homogeneity and normality of data distribution are found, repeated measures analysis of variance (ANOVA) and ANOVA with Bonferroni post hoc statistics will be used to analyze within and between groups. The Friedman test and Kruskal-Wallis test will be used when initial homogeneity but not normality of data distribution is found. If the initial homogeneity is not found, a linear mixed model will be adjusted for the baseline value. Adverse events in each group will be documented as percentage for safety assessments using the chi-square test or Fisher's exact test. The statistical significance is defined as $P<0.05$, and the $95 \%$ confidence interval will be reported.

\section{Sample size calculation}

The rate of the expectation of efficacy in the Tuina group is $95 \%$ and in the health care education group is $85 \%$, based on the literature. The sample size was calculated with a significance level of 0.05 and power of 0.80 , which also considers a maximum dropout tolerance of $10 \%$. Ultimately, 160 patients are needed for the trial, with 80 for each group.

\section{Quality control}

With the management of the steering committee, quality control will be conducted during the processing of the trial. Professional trial method and regular monitoring technique should be trained before the researchers participate in the trial, which can ensure the consistency of methods. The steering committee and ethics committee should be informed if the study protocol is modified or corrected.

\section{Discussion}

A survey showed that low back pain has a high prevalence rate across the lifespan, which seriously affects quality of life. In addition, the medical cost is a leading contributor to disease burden wordwide [4, 38, 39]. Systematic reviews of risk factors for low back pain have suggested that the rising risk of back pain episodes is related to both physical and psychological risk factors [40]. It can be caused by prolonged standing and lifting heavy weights, an unhealthy lifestyle such as smoking and obesity, experiencing distress, and the expectations that pain indicates bodily harm or injury. So, targeted health education in primary care is necessary and has been shown to provide long-term reassurance for patients with low back pain [23].

Tuina therapy belongs to the key noninvasive therapies of traditional Chinese medicine, which is based on the theory of meridians and acupoints and modern anatomy. It takes advantage of the hands of the therapist to manipulate the surface of the patient, including relaxation techniques, such as pressing, kneading, and pushing, and some adjustment manipulations, such as pulling and shaking. The Huangdi Inner Classic Pain Theory states that "when your body feels pain, it means the balance in your body is disrupted." Conversely, you will not feel any discomfort when your body's qi is flowing smoothly through the meridians. Blood stagnation is caused by the stagnation of qi, which can lead to phlegm pattern as the process of the disease. The most obvious manifestation is characterized by stabbing pain in a fixed location, usually aggravated at night. Tuina can help the patient achieve a physiological balance of "blood and qi" by manipulation of "restrict bone and harmonize joints." It has been found that patients with low back pain have a symptom that the muscle tension increases with local inflammatory stimulation and even continues to sputum in some biomechanical studies. Tuina is used to loosen muscle adhesions and significantly improves muscle strength of patients with low back pain. It can relieve the pathological fatigue state of the muscles, harmonize the coordinated balance function of the flexors and extensors, and finally restore a physiological balance of muscles and bones [41]. In previous studies, a rat model was established to demonstrate that Tuina could reduce pain sensitivity and peripheral nociceptive peripheral Cfiber activity in rats and could accelerate the repair peripheral nerve injury $[42,43]$.

However, the efficacy and safety of Tuina therapy requires further clinical proof. This trial is designed as a clinical randomized controlled trial to compare the efficacy of Tuina and health care education in patients with low back pain. The ODI, VAS, and SF-36 are being used as the outcome measurements, which can evaluate the efficacy of relieving pain and improve the quality of life 
with the interventions. This protocol intends to provide a more powerful evidence-based proof for Tuina therapy in patients with low back pain.

\section{Study limitations}

There is an inevitable limitation that it is difficult to control the methodology of blinding during the physical intervention. In this study, Tuina and health care education are different forms of physical therapy, which cannot be blinded for the participants and therapists. Therefore, the blinding quality should be monitored to control the questionnaire measurement credibility, which is composed of the assessors, the administrators, and the data analysts.

\section{Trial status}

This trial is still in the process of recruiting process. Participant recruitment started in June 2019 and will end in June 2020. This trial was registered in Chinese Clinical Trial Registry on 23 April 2019. The registration number is ChiCTR1900022656.

\section{Abbreviations}

ANOVA: Analysis of variance; CRF: Case report form; ICD11: International Classification of Diseases, 11th Revision; ODI: Oswestry Disability Index; SF36: 36-Item Short Form Health Survey; VAS: Visual analogue scale

\section{Acknowledgements}

Not applicable.

\section{Authors' contributions}

SZ planned the study protocol and drafted the manuscript. LK planned the study protocol and revised the manuscript. QZ carried out the Tuina and health care education interventions of patients. ZW recruited and screened eligible participants in the outpatient department. MF managed the study. WS was the study coordinator. $\mathrm{HJ}$ recruited and screened eligible patients in the inpatient department. YC participated in designing the outcome measurements and assessing the outcomes. SX participated in designing the trial and helped to prepare the manuscript. GG participated in assessing treatment efficacy and collected clinical data. XZ participated in the critical revision of the manuscript. ZL was responsible for generating and distributing the random numbers. All authors read and approved the final manuscript.

\section{Funding}

This study is supported financially by the project of Shenkang Three-Year Action "Clinical Skills Enhancement Project for Major Diseases" (16CR1023A), National Key Clinical Difficult Diseases Clinical Collaborative Pilot Construction Project - Osteoarticular Degenerative Disease (ZY[2018-2020]-FWTX-2005), Shanghai Further accelerate the 3-year action plan for the development of Chinese medicine (ZY [2018-2020]-CCCX-2004-02), Shanghai Health and Family Planning Commission Chinese Medicine Research Project (2018LP040), Shanghai Health and Family Planning System Outstanding Young Medical Talents (2018YQ004), China Association of Chinese Medicine Youth Enrollment Talent Project (QNRC2-A01), Talent Development Program in Shanghai (2019). The funders had no role in the design of the study; analysis, collection, and interpretation of the data; or the writing and decision for publication of the manuscript.

\section{Ethics approval and consent to participate}

Ethics approval was requested from and granted by the ethics committee of Yueyang Hospital of Integrated Traditional Chinese and Western Medicine, which is affiliated with Shanghai University of Traditional Chinese Medicine (reference 2019-035). Informed consent will be obtained from all study participants before any data collection by the clinical trial communicator. All participants will provide their consent in writing. Nobody except the investigators have access to the final data.

\section{Consent for publication}

This section is not applicable, because no personal information is provided in this article.

\section{Competing interests}

The authors declare that they have no competing interests.

\section{Author details}

${ }^{1} Y u e y a n g$ Hospital of Integrated Traditional Chinese and Western Medicine, Shanghai University of Traditional Chinese Medicine, Shanghai 200437, China. ${ }^{2}$ Institute of Tuina, Shanghai Institute of Traditional Chinese Medicine,

Shanghai 200437, China. ${ }^{3}$ School of Acupuncture-Moxibustion and Tuina,

Shanghai University of Traditional Chinese Medicine, Shanghai 201203, China.

Received: 16 July 2019 Accepted: 21 February 2020

Published online: 17 March 2020

\section{References}

1. Buchbinder R, Hartvigsen J, Dan C, et al. What low back pain is and why we need to pay attention. Lancet. 2018;391(10137):2356-67.

2. Hoy D, Bain C, Williams G, et al. A systematic review of the global prevalence of low back pain. Arthritis Rheum. 2012;64(6):2028-37.

3. Yiengprugsawan V, Hoy D, Buchbinder R, Bain C, Seubsman SA, Sleigh AC. Low back pain and limitations of daily living in Asia: longitudinal findings in the Thai cohort study. BMC Musculoskelet Disord. 2017;18:19.

4. Jackson T, Chen H, lezzi T, Yee M, Chen F. Prevalence and correlates of chronic pain in a random population study of adults in Chongqing, China. Clin J Pain. 2014;30(4):346-52.

5. Henschke N, Maher CG, Refshauge KM, et al. Prevalence of and screening for serious spinal pathology in patients presenting to primary care settings with acute low back pain. Arthritis Rheum. 2009;60(10):3072-80.

6. Smith BH, Fors EA, Korwisi B, et al. The IASP classification of chronic pain for ICD-11: applicability in primary care. Pain. 2019;160(1):83-7.

7. Hoy D, March L, Brooks P, et al. The global burden of low back pain: estimates from the Global Burden of Disease 2010 study. Ann Rheum Dis. 2014;73(6):968-74

8. Linton SJ, Boersma K. Early identification of patients at risk of developing a persistent back problem: the predictive validity of the Orebro Musculoskeletal Pain Questionnaire. Clin J Pain. 2003;19(2):80-6.

9. Hill JC, Dunn KM, Lewis M, et al. A primary care back pain screening tool: identifying patient subgroups for initial treatment. Arthritis Rheum. 2008; 59(5):632-41.

10. Dowell D, Haegerich TM, Chou R. CDC guideline for prescribing opioids for chronic pain-United States, 2016. JAMA. 2016:315(15):1624-45.

11. Gunter BR, Butler KA, Wallace RL, Smith SM, Harirforoosh S. Non-steroidal anti-inflammatory drug-induced cardiovascular adverse events: a metaanalysis. J Clin Pharm Ther. 2017;42(1):27-38.

12. Deyo RA, Smith DH, Johnson ES, et al. Opioids for back pain patients: primary care prescribing patterns and use of services. J Am Board Fam Med. 2011;24(6):717-27.

13. Kanno H, Aizawa T, Hahimoto K, Itoi E. Minimally invasive discectomy for lumbar disc herniation: current concepts, surgical techniques, and outcomes. Int Orthop. 2019;43(4):917-22.

14. Bernstein IA, Malik Q, Carville S, Ward S. Low back pain and sciatica: summary of NICE guidance. BMJ. 2017;356:6748.

15. Qaseem A, Wilt TJ, McLean RM, Forciea MA. Noninvasive treatments for acute, subacute, and chronic low back pain: a clinical practice guideline from the American College of Physicians. Ann Intern Med. 2017;166(7):514-30.

16. Epping R, Verhagen AP, Hoebink EA, Rooker S, Scholten-Peeters G. The diagnostic accuracy and test-retest reliability of the Dutch PainDETECT and the DN4 screening tools for neuropathic pain in patients with suspected cervical or lumbar radiculopathy. Musculoskelet Sci Pract. 2017;30:72-9.

17. Foster NE, Anema JR, Cherkin D, et al. Prevention and treatment of low back pain: evidence, challenges, and promising directions. Lancet. 2018; 391(10137):2368-83.

18. Institute of Medicine, Committee on Advancing Pain Research, Care, and Education. Relieving pain in America: a blueprint for transforming 
prevention, care, education, and research. Washington, DC: National Academies Press; 2011.

19. Delitto A, George SZ, Van Dillen L, et al. Low back pain. J Orthop Sports Phys Ther. 2012;42(4):A1-57.

20. Moseley L. Combined physiotherapy and education is efficacious for chronic low back pain. Aust J Physiother. 2002;48(4):297-302

21. Moseley L. Unraveling the barriers to reconceptualization of the problem in chronic pain: the actual and perceived ability of patients and health professionals to understand the neurophysiology. J Pain. 2003;4(4):184-9.

22. Wijma AJ, Speksnijder CM, Crom-Ottens AF, et al. What is important in transdisciplinary pain neuroscience education? A qualitative study. Disabil Rehabil. 2018;40(18):2181-91.

23. Traeger AC, Hubscher M, Henschke N, Moseley GL, Lee H, McAuley JH. Effect of primary care-based education on reassurance in patients with acute low back pain: systematic review and meta-analysis. JAMA Intern Med. 2015;175(5):733-43.

24. Saragiotto BT, Maher CG, Traeger AC, Li Q, McAuley JH. Dispelling the myth that chronic pain is unresponsive to treatment. Br J Sports Med. 2017;51(13): 986-8.

25. Hidalgo B, Hall T, Bossert J, Dugeny A, Cagnie B, Pitance L. The efficacy of manual therapy and exercise for treating non-specific neck pain: a systematic review. J Back Musculoskelet Rehabil. 2018;30(6):1149-69.

26. Kumar S, Beaton $K$, Hughes $T$. The effectiveness of massage therapy for the treatment of nonspecific low back pain: a systematic review of systematic reviews. Int J Gen Med. 2013;6:733-41.

27. Ruddock JK, Sallis H, Ness A, Perry RE. Spinal manipulation vs sham manipulation for nonspecific low back pain: a systematic review and metaanalysis. J Chiropr Med. 2016;15(3):165-83.

28. Pach D, Piper M, Lotz F, et al. Effectiveness and cost-effectiveness of Tuina for chronic neck pain: a randomized controlled trial comparing Tuina with a no-intervention waiting list. J Altern Complement Med. 2018;24(3):231-7.

29. Du S, Hu L, Dong J, et al. Self-management program for chronic low back pain: a systematic review and meta-analysis. Patient Educ Couns. 2017; 100(1):37-49.

30. Engers A, Jellema $P$, Wensing M, van der Windt DA, Grol R, van Tulder MW. Individual patient education for low back pain. Cochrane Database Syst Rev. 2008;1:D4057

31. Deng $Q$, Wu Y, He C, Sun Y, Ma G, Tian Y. Efficacy differences between different position in patients treated with electroacupuncture for lumbar herniated disc [in Chinese]. Zhongguo Zhen Jiu. 2016;36(7):689-93.

32. Zai FL, Wu RL, Zheng MF, Guo LY. Warming needle moxibustion relieves symptoms of lumbar disc herniation patients and upregulates plasma betaendorphin [in Chinese]. Zhen Ci Yan Jiu. 2018;43(8):512-5.

33. Zhang K, Xu Y, Ding S, Hong S, Zhao X, Guo Y. Literature study for acupoint selection rule of rheumatoid arthritis treated with acupuncture [in Chinese]. Zhongguo Zhen Jiu. 2017;37(2):221-4.

34. Chen D, Yang G, Wang F, Qi W. Discussing the relationship among the ashi point, tender point and myofascial trigger point [in Chinese]. Zhongguo Zhen Jiu. 2017;37(2):212-4.

35. Mo Z, Zhang R, Chen J, Shu X, Shujie T. Comparison between oblique pulling spinal manipulation and other treatments for lumbar disc herniation: a systematic review and meta-analysis. J Manip Physiol Ther. 2018;41(9):771-9.

36. Brodke DS, Goz V, Voss MW, Lawrence BD, Spiker WR, Hung M. PROMIS PF CAT outperforms the ODI and SF-36 Physical Function domain in spine patients. Spine (Phila Pa 1976). 2017:42(12):921-9.

37. Faiz KW. VAS - visual analog scale [in Norwegian]. Tidsskr Nor Laegeforen. 2014;134(3):323.

38. Garcia JB, Hernandez-Castro JJ, Nunez RG, et al. Prevalence of low back pain in Latin America: a systematic literature review. Pain Physician. 2014;17(5): 379-91.

39. Dagenais S, Caro J, Haldeman S. A systematic review of low back pain cost of illness studies in the United States and internationally. Spine J. 2008;8(1): 8-20.

40. Taylor JB, Goode AP, George SZ, Cook CE. Incidence and risk factors for firsttime incident low back pain: a systematic review and meta-analysis. Spine J. 2014;14(10):2299-319.

41. Nan Z, Min F, Zhu Q, et al. Evaluation of biomechanical properties of lumbar and dorsi extensor muscles in the treatment of lumbar disc herniation by massage manipulation. Chin J Trad Chin Med. 2012;27(3):562-6.

42. Jiang $S$, Zhang $H$, Fang $M$, et al. Analgesic effects of Chinese Tuina massage in a rat model of pain. Exp Ther Med. 2016;11(4):1367-74.
43. Pan F, Yu TY, Wong S, et al. Chinese Tuina downregulates the elevated levels of tissue plasminogen activator in sciatic nerve injured SpragueDawley rats. Chin J Integr Med. 2017;23(8):617-24.

\section{Publisher's Note}

Springer Nature remains neutral with regard to jurisdictional claims in published maps and institutional affiliations.
Ready to submit your research? Choose BMC and benefit from:

- fast, convenient online submission

- thorough peer review by experienced researchers in your field

- rapid publication on acceptance

- support for research data, including large and complex data types

- gold Open Access which fosters wider collaboration and increased citations

- maximum visibility for your research: over $100 \mathrm{M}$ website views per year

At BMC, research is always in progress.

Learn more biomedcentral.com/submissions 